\title{
Deformation field interaction in sequential circular indentation of a strain hardening material
}

\section{Zhiyu Wang, Tejas G. Murthy \& Christopher Saldana}

To cite this article: Zhiyu Wang, Tejas G. Murthy \& Christopher Saldana (2019): Deformation field interaction in sequential circular indentation of a strain hardening material, Philosophical Magazine, DOI: $10.1080 / 14786435.2019 .1580396$

To link to this article: https://doi.org/10.1080/14786435.2019.1580396

$$
\text { 曲 Published online: } 27 \text { Feb } 2019 .
$$

\section{Submit your article to this journal $\widetilde{ }$}

Џll Article views: 6

View Crossmark data ¿ 


\title{
Deformation field interaction in sequential circular indentation of a strain hardening material
}

\author{
Zhiyu Wang ${ }^{\mathrm{a}}$, Tejas G. Murthy ${ }^{\mathrm{b}}$ and Christopher Saldana ${ }^{\mathrm{a}}$ \\ aThe George W. Woodruff School of Mechanical Engineering, Georgia Institute of Technology, Atlanta, \\ Georgia; ${ }^{b}$ Department of Civil Engineering, Indian Institute of Science, Bangalore, India
}

\begin{abstract}
An experimental study was made to characterise and model the deformation field in sequential circular indentation of a model strain hardening material. Digital image correlation was used to measure the evolving subsurface deformation field in terms of displacement, strain rate and strain as a function of indentation spacing and depth. These measurements were used to validate a finite element model for complementary simulations. The results identify relationships between sequential indentation parameters and overlap of subsurface strain distributions, maximum subsurface strains and indentation loads. Maximum strain and the degree of strain field overlap in the deformed subsurface were maximised when the ratio of indentation spacing $(\mathrm{S})$ to projected indentation contact length (L) was approximately $S / L=[1.1,1.2]$. Also discussed are the implications for understanding process-scale considerations for indentation-based mechanical surface treatments, including energy dissipation and relationship of surface coverage measures to subsurface strain overlap. Relative differences in energy expended were found for conditions that produce similar levels of subsurface plastic strain and strain field overlap. Finally, the role of sequential indentation parameters on strain path changes and path reversals in the deformed subsurface is investigated and discussed in the context of heterogeneous mechanics and corresponding effects on subsurface microstructure evolution.
\end{abstract}

\section{ARTICLE HISTORY}

Received 2 August 2018

Accepted 3 February 2019

\section{KEYWORDS}

Indentation; digital image correlation; experimental; finite-element modelling

\section{Introduction}

Deformation occurring in sequential indentation loading is critical for understanding the governing mechanics associated with mechanical surface treatment methods. It has been well investigated that the surface integrity of metallic materials can be enhanced by using appropriate mechanical surface modification techniques. More recently, significant interest has been found in the use of these approaches to achieve superior functional performance through the fabrication 
of gradient microstructures due to surface severe plastic deformation (S2PD) [1]. In these S2PD methods, near surface layers are subject to high plastic strains that decay into the subsurface, this yielding a gradient of grain refinement dependent on the strains imposed at the surface. These gradient materials have been shown to exhibit improved resistance to wear [2], fatigue [3,4] and corrosion [5,6], as well as a superior combination of ductility and strength $[7,8]$. S2PD approaches based on surface mechanical attrition treatment (SMAT) [9-11] and ultrasonic impact treatment (UIT) [6] rely on repeated surface impact to impart graded strain levels; these process configurations have gained significant attention due to their inherent high controllability and compatibility with complex part geometries [12]. While similar to conventional air blast shot peening (ABSP) in their general principle [13], discrepancies between these methods exist in terms of the size of the tool geometry (i.e. diameter), as well as the relative spacing (i.e. overlap), depth and especially direction of loading with respect to the surface. Selection of these parameters requires consideration of optimal treatment schedules to achieve specific levels of maximum strain as well as subsurface coverage in terms of the overlap of individual strain fields from unit-scale deformations.

The unit deformation field under multiple indentation-type loading events has been studied in the context of phenomena associated with sequential indentation and simultaneous co-indentation. Understanding of deformation in sequential indentation loading is important for the effective selection of materials testing parameters that involve indentation-based assessments of hardness and strength. Critical spacing of indents is dependent on the relative size of the elastic-plastic zone underneath a single indent, where this spacing is generally set large enough to prevent substantial overlap of the associated subsurface plastic zones. Samuels and Mulhearn have shown that a nominal indentation spacing of $2.7 d_{p}$, where $d_{p}$ is the indentation depth using a pyramid indenter, is sufficient to ensure less than $5 \%$ isostrain overlap in successive indentations [14]. With this as a basis for determination of indentation spacing, a host of other similar studies that have established the size of the elastic-plastic zone can inform similar estimates of minimum indentation spacing based on indenter geometry and workpiece material. In addition to the sequential indentation problem, the effects of multiple indentation-type loading on subsurface deformation have been studied through simultaneous co-indentation configurations wherein a surface is deformed concurrently by a pair of indenters set at controlled relative spacing [15]. The effect of indenter separation length was investigated in terms of residual stress components and the development of subsurface plastic zones. While these studies have guided the development of finite element (FE) models for process scale simulations [16-18], they inherently have relied on post mortem assessments for validation of the evolving plastic field and have not provided a framework validated using measurements of the evolving plastic zones present due to multiple indentations. 
Fundamental understanding of the evolving deformation field in indentation loading is possible using in-situ experimental assessments [19-21], this complemented by validated FE models $[17,22]$. These in-situ methods have been used to characterise the subsurface deformation field in the indentation of surfaces under various tool geometries. These studies have revealed and/or verified key features of the deformation field, such as the formation of material stagnation zones (e.g. dead zones) under penetration due to rigid flat punches and circular indenters [23]. Further, these in-situ approaches have been useful for calibration of dead zone and plastic zone formulations for expanding cavity models to predict the state of the deformed subsurface $[19,24]$. The objective of present study is to elucidate an understanding of the effects of controllable parameters in sequential indentation of a model strain hardening material on size of the overlapping plastic zones as well as characteristics of the overall strain field in terms of gradient and maximum strain levels imposed. For this purpose, oxygen-free high-conductivity (OFHC) $\mathrm{Cu}$ was used as a model material as it is a conventional representative of a wide range of moderate stacking fault energy face centred cubic material systems. Indentation studies were carried out at a macroscopic length scale and in-situ mapping of material flow at high spatial resolution were facilitated using a digital imaging platform to quantify the deformation field during two sequential circular indentions under planestrain conditions. An FE model was validated and the effect of indentation depth and separation length on the evolution of strain components and indentation load was investigated. From the present results, empirical frameworks for control of subsurface strains and strain field overlap are elucidated. Further, the relative energy efficiencies and strain path changes due to specific combinations of indentation spacing and depth parameters used during indentation-type processing are evaluated.

\section{Experimental methods}

Figure 1(a) shows a schematic of the experimental configuration used in the present study. Experiments were carried out under plane-strain conditions

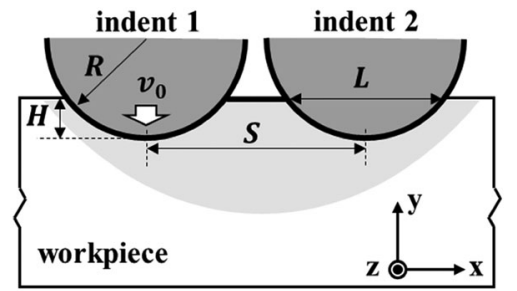

(a)

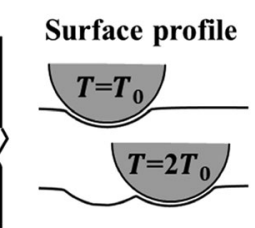

(b)

Figure 1. Schematic representation of (a) sequential indentation configuration and (b) surface profiles yielded from single and two indentations. 
ensured by planar constraint of the imaged work surface by a tempered glass plate and constraint of the opposing surface by a steel backer plate. In this configuration, a series of indents were carried out sequentially on a workpiece. Each indentation in the sequence was conducted to the same final depth of indentation $(H)$, rate of indentation $\left(v_{0}\right)$ and processing time $\left(T_{0}\right)$. To provide relative positioning of each indent, the workpiece was shifted in the lateral direction to yield an effective centreline-to-centreline spacing length $(S)$. In the present study, the indenter was loaded with $H=1.5 \mathrm{~mm}, v_{0}=0.1 \mathrm{~mm} / \mathrm{s}, T_{0}=$ $15 \mathrm{~s}$ and $S=2.5 \mathrm{~mm}$. The projected contact length of the indenter with the workpiece $(L)$ is given by $L=2 \sqrt{R^{2}-(R-H)^{2}}$. The resulting surface profiles produced from the first and second indents are shown schematically in Figure 1(b). For this study, a circular indenter of radius $R=2.5 \mathrm{~mm}$ was constructed from tool steel by electrical discharge machining (EDM) and finished by polishing the indenter surface with 1000 grid $\mathrm{SiC}$ paper. OFHC $\mathrm{Cu}$ workpieces were machined into $25 \mathrm{~mm} \times 60 \mathrm{~mm} \times 6 \mathrm{~mm}$ blocks pre-annealed at $600^{\circ} \mathrm{C}$ for $2 \mathrm{~h}$.

Quantitative analysis of the deformation was conducted by in situ imaging of the surface deformation. A uniaxial testing machine (Instron 5982) was used in displacement control mode and the material flow was recorded using a highspeed camera (PCO Dimax). To facilitate image tracking, asperities on the imaged workpiece surface were imparted by abrasion with a 600 grit $\mathrm{SiC}$ pad prior to the indentation tests. The overall field of view was $2000 \times 2000$ pixels with a spatial resolution of $5 \mu \mathrm{m} /$ pixel. Local displacement fields from between each pair of consecutive images were determined using a digital image correlation (DIC) algorithm. Subpixel accuracy for these displacement fields were achieved by using a Newton-Raphson algorithm, wherein the initial guess is estimated by fast Fourier transform (FFT) based correlation [25]. The strain rate tensor $\dot{\varepsilon}_{i j}=(1 / 2)\left(\left(\partial v_{i} / \partial x_{j}\right)+\left(\partial v_{j} / \partial x_{i}\right)\right)$ was obtained by differentiation of the displacement field. The effective plastic strain field was determined by temporal integration of local incremental strain fields along path trajectories, defined by $\bar{\varepsilon}=\sqrt{(2 / 3) \dot{\varepsilon}_{i j} \dot{\varepsilon}_{i j}} \mathrm{~d} t$. A relative strain rate measurement error of less than 0.01 was found for the measured displacement by evaluation of simulated displacements of real marker fields. Correspondingly, a total strain measurement error of 0.1 was found for the images obtained. This strain and strain rate error is associated with DIC analysis of the real marker field, which is resultant from the magnification used and surface condition. The relative error corresponding to ideal marker fields typically falls below 0.01 in this regard.

\section{Simulation methods}

Deformation in sequential indentation was simulated as a series of indents using FE analysis (ABAQUS/Explicit). The indenter was modelled as a discrete rigid body and the workpiece was modelled as an isothermal body using four-node 


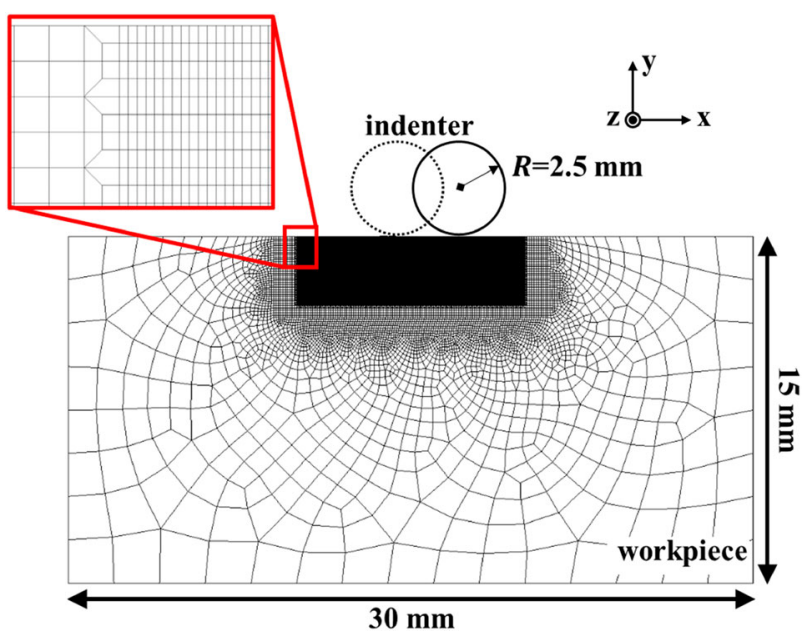

Figure 2. Finite element (FE) model of the workpiece.

and three-node bilinear, reduced integration, and plane-strain elements as shown in Figure 2. The isothermal state of the workpiece was justified considering the low loading rates used in the experimental studies. The Johnson-Cook material model was used with

$$
\sigma=\left[A+\left(B \bar{\varepsilon}^{p l}\right)^{n}\right]\left[1+C \times \log \left(\frac{\dot{\bar{\varepsilon}}^{p l}}{\dot{\varepsilon}_{0}}\right)\right]
$$

where $\left[A, B, C, \dot{\varepsilon}_{0}, n\right]=\left[90,292 \mathrm{MPa}, 0.025,1 \mathrm{~s}^{-1}, 0.31\right]$ for OFHC Cu [26].

The indenter was vertically translated into the specimen surface by displacement control with a constant loading rate of $0.1 \mathrm{~mm} / \mathrm{s}$, retracted and then displaced in the lateral direction with a certain spacing length and repeated for the second indent. The contact was defined as node-to-node contact of the master and slave surfaces with Coulomb friction coefficient of $\mu=0.2$. This friction coefficient was determined by simulation trials that showed agreement with deformation fields of the experimental results. The accuracy of the simulation was assessed by comparing the simulated effective plastic strain and indentation load with the DIC-based measurements, as in the ensuing. After validating the simulation framework, a series of parametric simulations were conducted with $H / R=[0.1,0.2,0.3,0.4,0.5]$, and $S / R=[0.1,0.2,0.5,1.0,2.0,3.0]$.

\section{Results}

The deformation occurring in multiple indentations on the OFHC workpiece was measured using in-situ DIC. Figure 3 shows optical images taken from a series of indents carried out up to a final depth of $H / R=0.3$ and with a relative spacing of $S / R=1.0$. The optical image frames in Figure 3(a) and (d) show the 

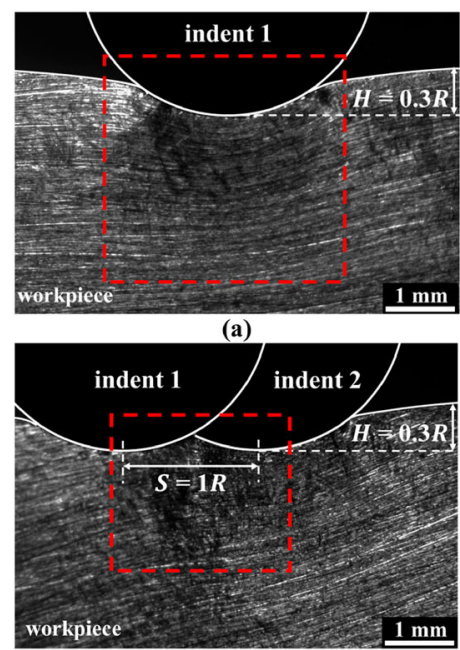

(d)

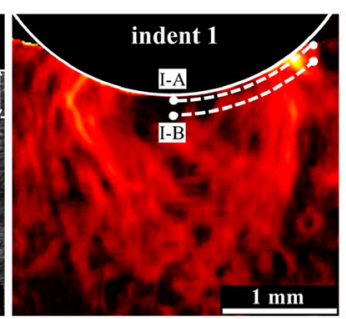

(b)

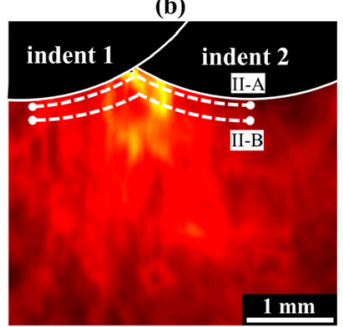

(e)

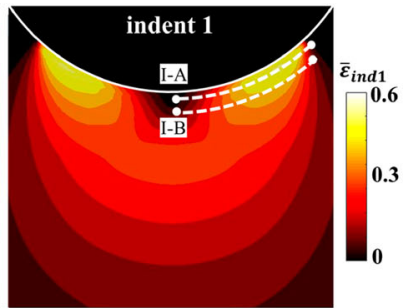

(c)

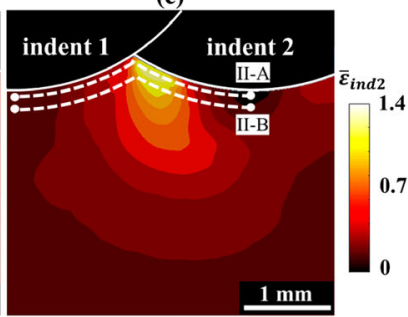

(f)

Figure 3. (a) Optical image of final deformed workpiece, (b) experimental and (c) FE-predicted distribution of $\bar{\varepsilon}$ after a single indent at $H / R=0.3$. (d) Optical image of final deformed workpiece, (e) experimental and (f) FE-predicted distribution of $\bar{\varepsilon}$ after the second indent at $S / R=1.0$.

state of workpiece at the final indentation depth for the first indent and second indent, respectively. The corresponding effective strain fields for these conditions are provided in Figure 3(b) and (e). As is seen in the figures, the deformation occurring in the first indent is similar to that previously reported for circular indentation of OFHC $\mathrm{Cu}$ in Ref. [19]. As reported, a hemispherical region of material displaces with the indenter as it penetrates into the workpiece. At the boundary of this dead zone, a semi-circular banded region persists where the maximum effective strain at normalised indentation depths of $H / R=0.19$ and $H / R=0.33$ was $\bar{\varepsilon} \sim 0.23$ and $\bar{\varepsilon} \sim 0.34$, respectively [19]. It was also noted that at larger indentation depths, these bands extended to greater subsurface depths and become increasingly diffuse. In the present work, the depth of the plastic zone at an indentation depth of $H / R=0.3$ was $\sim 1.10 R$ and the lateral extent of this plastic zone was $\sim 1.45 R$. At greater subsurface depths, the effective strain falls below an appreciable level of $\bar{\varepsilon}<0.01$, wherein elastic deformation is likely more dominant. The deformation occurring during the second indent is also shown in Figure 3(e). From the deformation fields, a maximum plastic strain in the deformed subsurface $\bar{\varepsilon} \sim 0.6$ was obtained after the first indent and this was increased to $\bar{\varepsilon} \sim 1.3$ after the second indent.

From the effective strain fields, the plastic strain at each loading step was measured along pre-defined contours directly underneath the indenter (e.g. IA, I-B, II-A, II-B) and offset from the surface by $0.05 R$ and $0.3 R$, respectively, as is shown in Figure 3(b) and (e). The resulting distribution along these curves is summarised in Figure 4(a) and (b). The subsurface strain distributions along these curves for the first indent are summarised in Figure 4(a). The 


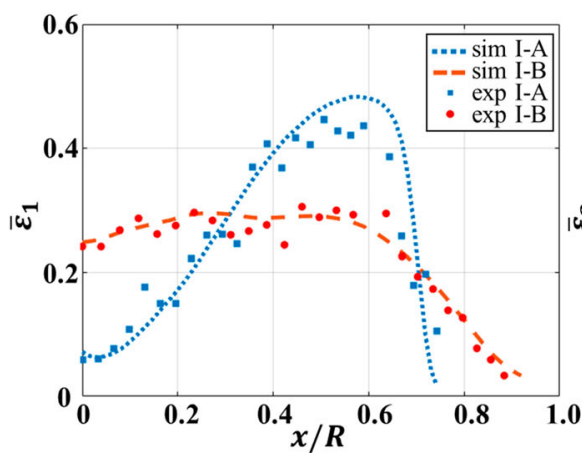

(a)

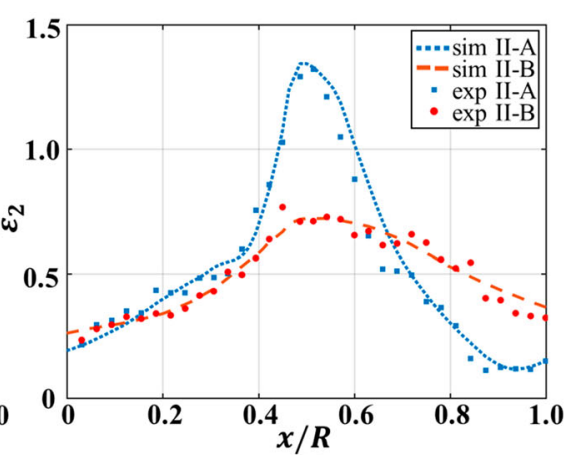

(b)

Figure 4. Comparison of effective plastic strain between DIC-obtained and FE-predicted results along contours I-A, I-B, II-A, and II-B after (a) single and (b) sequential indentation at $H / R=0.3$ and $S / R=1.0$.

effective strain along the contour I-A at $x / R=0.0$ (e.g. centreline of the first indent) was $\bar{\varepsilon} \sim 0.08$, which is lower than the corresponding strain of $\bar{\varepsilon} \sim 0.23$ at a similar position in contour I-B. This is expected as contour I-A is closer to the dead zone. The plastic strain along contour I-A reaches a maximum value of $\bar{\varepsilon} \sim 0.48$ at $x / R=0.5$ and rapidly decreases to $\bar{\varepsilon} \sim 0.05$ at $x / R=0.7$. Similarly, the plastic strain along contour I-B reaches the maximum value of $\bar{\varepsilon} \sim 0.30$ at $x / R=0.5$ and decreases to $\bar{\varepsilon} \sim 0.05$ at $x / R=0.8$. These observations are consistent with both the presence of a region of low strain (i.e. dead zone) near the indent centreline and a narrow high strain band formed toward the periphery of the indenter contact and flanking the dead zone.

The cumulative strain distribution resulting from the second indent along contours II-A and II-B are shown in Figure 4(b). For the second indent, this shows that the maximum induced effective strain of $\bar{\varepsilon} \sim 1.32$ occurs at $x / R=$ 0.5 for contour II-A, this compared to $\bar{\varepsilon} \sim 0.73$ for contour II-B. It should be noted that the strain in the dead zone region of the first indent was increased by the second indent in that the cumulative effective strain at $x / R=0.0$ was $\bar{\varepsilon} \sim 0.21$ and $\bar{\varepsilon} \sim 0.32$ for contours II-A and II-B, respectively. Figure 3(c) and (f) shows the FE-predicted distribution fields of effective plastic strain in the same regions reported above from the experimental measurements. The corresponding strain distributions are reported in Figure 4(a) and (b) and good agreement is found between the experimental measurements and the FE-based predictions. The experimental and predicted results in Figure 4 matched closely with $R^{2}=0.98$, this providing validation of the simulation framework.

Further validation of the simulation results was made by comparison of the indentation load to the experimental measurements. Figure 5 shows the comparison of the indentation load with respect to normalised indentation depth $H / R=[0.1,0.2,0.3,0.4,0.5]$ during the first indentation in the sequence. In the figure, the normalised indentation load from the FE model ranged from 


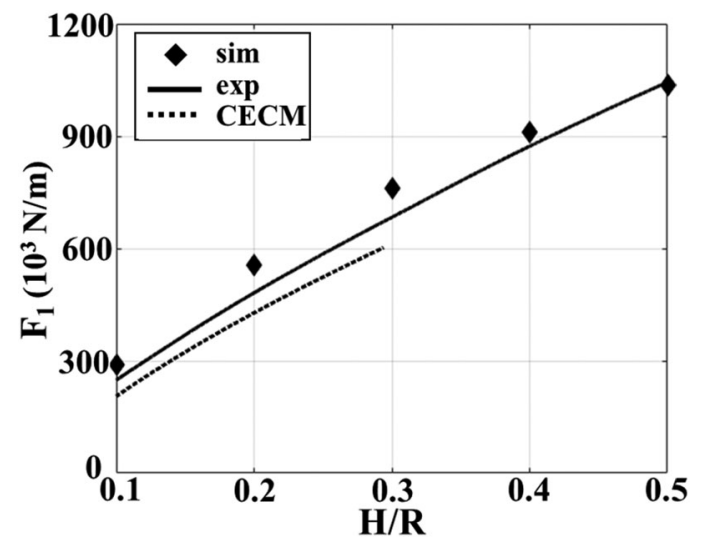

Figure 5. Indentation load with respect to normalised depth of indentation of $H / R$ during the first indentation.

$F_{1}=280 \times 10^{3} \mathrm{~N} / \mathrm{m}$ to $F_{1}=1130 \times 10^{3} \mathrm{~N} / \mathrm{m}$ and was close to the experimentally measured values obtained within less than $15 \%$ relative error. These data were also in good agreement with the load evolution predicted by a calibrated expanding cavity model (CECM) established in a prior study [19]. Comparing both models, the FE simulation provided a better estimation of indentation load compared to the analytical solution. It is also worthwhile to note that the CECM is not valid at larger normalised indentation depth of $H / R>0.3$ as assumptions regarding the growth of the internal expanding cavity are not valid in this regime.

After validation, a series of simulations were carried out to explore the role of controllable parameters in sequential indentation on the subsurface plastic zone. For this purpose, normalised indentation depths of $H / R=[0.2,0.3]$ and normalised indentation spacings of $S / R=[0.1,0.2,0.5,1.0,2.0,3.0]$ were evaluated. Figure 6 shows the result of the simulation results with Figure 6(a)-(f) at $H / R=0.2$ and Figure 6(g)-(l) at $H / R=0.3$. From the figure, it is clear that the normalised indentation spacing determines the overall nature of the

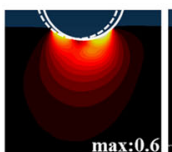

(a)

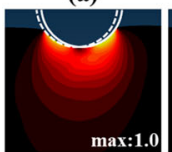

(g)

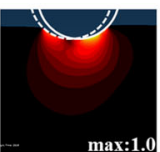

(b)

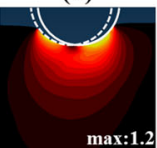

(h)

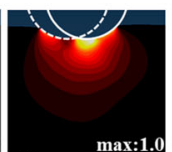

(c)

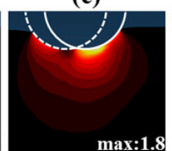

(i)

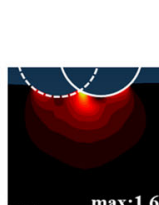

(d)

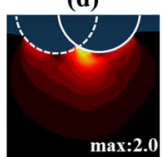

(j)

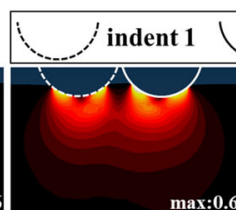

(e)

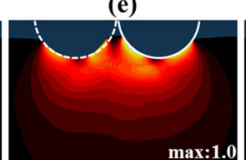

(k)
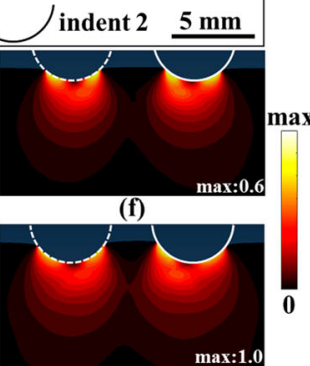

(1)

Figure 6. Effective plastic strain fields of subsurface regions from FE model with $S / R=[0.1,0.2$, $0.5,1.0,2.0,3.0]$ and (a)-(f) $H / R=0.2$, (g)-(l) $H / R=0.3$. 
subsurface deformation zone. At small values with $S / R<0.2$, as in Figure 6(a), (b), (g) and (h), the effective strain distributions after the second indentation were markedly similar to deformation fields reported for a single indent [19]. A noticeable dead zone region was produced underneath the indenter that featured low plastic strains with $\bar{\varepsilon}<0.1$. Additionally, a band of high strain rate and strain surrounded the dead zone and featured high effective strains with $\bar{\varepsilon}>0.5$. As the normalised indentation spacing was increased to $1.0 \geq S / R \geq 0.5$, as shown in Figure 6(c), (d), (i), and (j), regions of the deformed subsurface were exposed to strains with $\bar{\varepsilon}>1.0$, primarily those located between the two indents. In this case, the maximum effective plastic strain in the deformed subsurface was nearly double that resultant after a single indent. At normalised indentation spacing with $S / R \geq 2.0$, as in Figure 6(e), (f), (k), and (l), the individual deformation fields associated with each indent were more clearly visible due to the increased separation. Further, at the largest normalised indentation spacing of $S / R=3.0$, the maximum plastic strain in the deformed subsurface was equivalent to that seen for the case of a single indent and/or a closelyspaced set of sequential intents, as in Figure 6(a) and (g).

\section{Discussion}

The present study investigated the effects of process parameters in sequential indentation on mechanical responses of a model strain hardening material in terms of the subsurface deformation field and indentation load. These effects were studied using a validated simulation model. In the context of controllability of indentation-based methods for imparting controlled subsurface strain distributions, the present results have utility for understanding the relationship between surface and subsurface coverage, energy dissipation and role of strain path changes in heterogeneous surface deformation. Each of these implications are discussed in the ensuing.

From the present results, subsurface deformation in indentation-based $S^{2} \mathrm{PD}$ is controllable by appropriate selection of indentation depth and spacing parameters. Measures of interest to quantify severity and homogeneity of the deformation include the maximum strain in the deformed subsurface and the degree of deformation overlap (e.g. subsurface coverage) in the resulting strain fields, respectively. In order to quantify the severity of the deformation, the normalised maximum effective plastic strain in the deformed subsurface for the first indentation, $\bar{\varepsilon}_{1 \_ \text {max }} / \bar{\varepsilon}_{0.5 R}$, was characterised with respect to normalised indentation depth $H / R$, where $\bar{\varepsilon}_{0.5 R}$ refers to the maximum effective plastic strain with $H / R=0.5$. The normalised maximum strain increases from $\bar{\varepsilon}_{1_{-} \max } / \bar{\varepsilon}_{0.5 R}=$ 0.45 to $\bar{\varepsilon}_{1 \_ \text {max }} / \bar{\varepsilon}_{0.5 R}=1.0$ from $H / R=0.1$ to $H / R=0.5$, as shown in Figure 7 (a). The normalised maximum effective strain for the second indentation, $\bar{\varepsilon}_{2 \_ \text {max }} / \bar{\varepsilon}_{0.5 R}$, depends on both the normalised indentation spacing $S / R$ and the normalised indentation depth $H / R$, as shown in Figure $7(\mathrm{~b})$. Of particular 


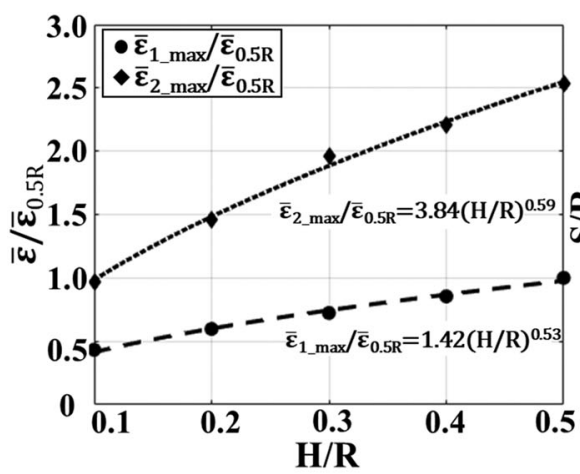

(a)

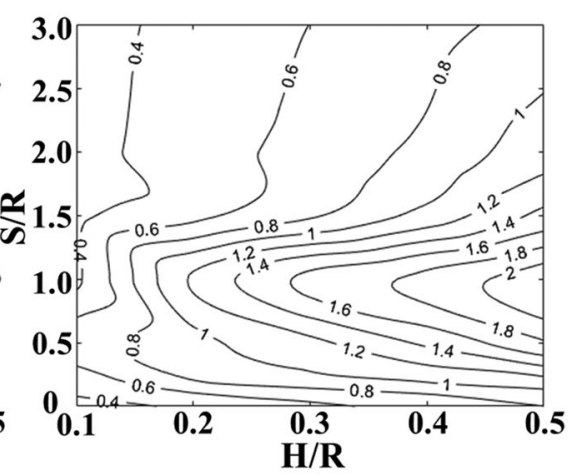

(b)

Figure 7. Normalised maximum effective plastic strain with respect to (a) $H / R$ for single and sequential indentation and (b) $H / R$ and $S / R$ for sequential indentation.

interest is the maximum effective strain for each value of normalised indentation depth $H / R$ across all values of normalised indentation spacing $S / R$. These results are shown in Figure 7 (a) for $\bar{\varepsilon}_{2_{-} \max } / \bar{\varepsilon}_{0.5 R}$, where the normalised maximum strain increases from $\bar{\varepsilon}_{2_{-} \max } / \bar{\varepsilon}_{0.5 R}=1.0$ to $\bar{\varepsilon}_{2_{-} \max } / \bar{\varepsilon}_{0.5 R}=2.5$ from $H / R=0.1$ to $H / R=0.5$. The peak strain at each stage followed a relationship in the form: $\bar{\varepsilon}_{\max } / \bar{\varepsilon}_{0.5 R}=C(H / R)^{n}$, where $(C, n)=(1.42,0.53)$ for the first indent and $(C, n)=(3.84,0.59)$ for the second indent. The $R$-squared value for these fits were both 0.99 .

To determine the role of normalised deformation parameters $H / R$ and $S / R$ in affecting subsurface deformation coverage, the subsurface effective strain fields were characterised for degree of strain field overlap. Figure 8 shows the size of the deformation-affected region across a range of indents from $S / R=[0.1,0.2$, $0.5,1.0,1.5,2.0]$ at $H / R=0.3$. In this figure, individual nodes were categorised into two classes dependent on whether the respective total cumulative strain of each node was primarily determined by the first indentation $\left(\bar{\varepsilon}_{1} \geq 0.1, \Delta \bar{\varepsilon}<0.1\right)$ or by both indentations $\left(\bar{\varepsilon}_{1} \geq 0.1, \Delta \bar{\varepsilon} \geq 0.1\right)$, where $\bar{\varepsilon}_{1}$ is the effective strain due to the first indent and $\Delta \bar{\varepsilon}$ is the incremental effective strain associated with the second indent. In the below discussion, the latter of these two regions is referred to as the subsurface overlap region. The threshold of $\bar{\varepsilon} \geq 0.1$ is based on the minimum deformation level that has been observed to alter the microstructure in the subsurface of a $\mathrm{S}^{2} \mathrm{PD}$-processed copper materials [27]. From the figure, it is evident that the size of the subsurface overlap region is dependent on the indentation spacing length. At a normalised depth of indentation of $H / R=0.3$, the area of overlap region ranged from $0.25 \times$ $10^{-6} \mathrm{~m}^{2}$ at $S / R=0.1$ to the maximum value of $2.06 \times 10^{-6} \mathrm{~m}^{2}$ at $S / R=1.0$, and then gradually decreased to zero at $S / R=3.0$.

To understand the effect of normalised indentation depth $H / R$ on the size of the plastic zone, Figure 9(a) summarises the size of the subsurface area wherein a 


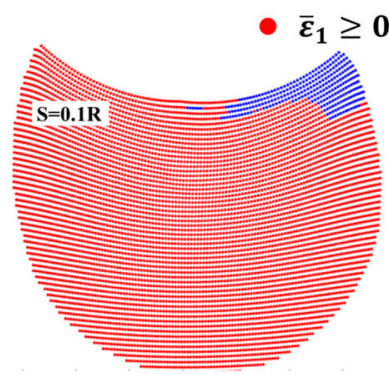

(a)

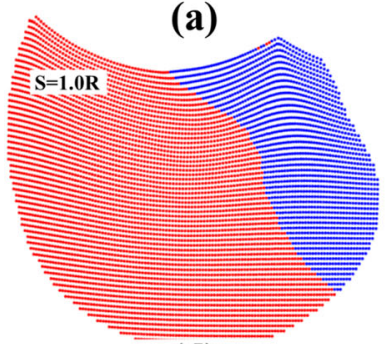

(d)

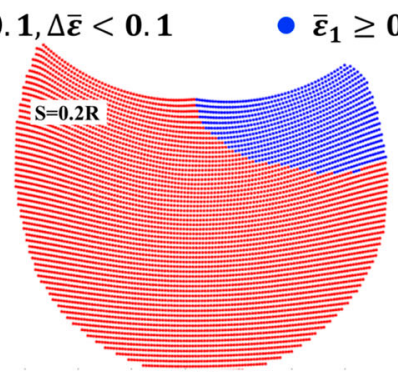

(b)

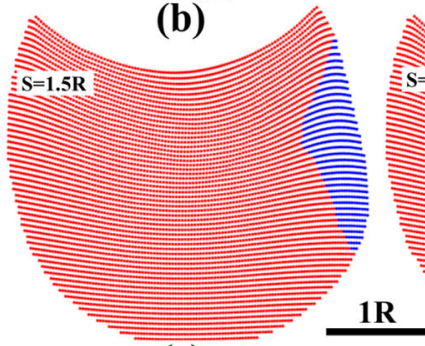

(e)
$0.1, \Delta \bar{\varepsilon} \geq 0.1$

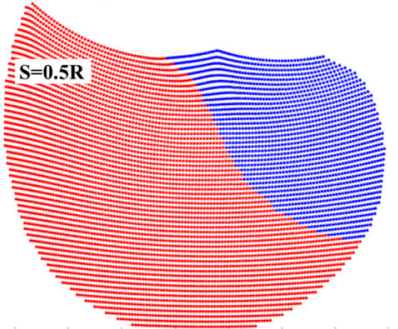

(c)

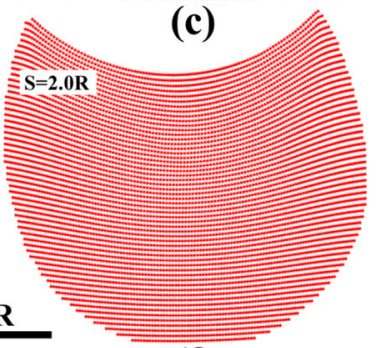

(f)

Figure 8. Deformation-affected region produced by sequential indentation configuration at normalised indentation depth of $H / R=0.3$. Red nodes indicate the regions only deformed by the first indent and blue nodes indicate the regions affected by both indentations.

threshold strain was achieved. As normalised indentation depth increases from $H / R=0.1$ to $H / R=0.5$, the normalised area of the plastic zone increased from $A_{1} / A_{0.5 R}=0.1$ to $A_{1} / A_{0.5 R}=1.0$, where $A_{0.5 R}$ refers to the area of region plastically deformed such that $\bar{\varepsilon}_{1} \geq 0.1$ with $H / R=0.5$. The size of the overlap region was also quantified as a function of normalised indentation depth $H / R$ where material in the overlap region $A_{2} / A_{0.5 R}$ satisfy $\bar{\varepsilon}_{1} \geq 0.1$ and $\Delta \bar{\varepsilon} \geq 0.1$. As was the case for normalised maximum effective strain, the size of the overlap region depends on both the normalised indentation spacing $S / R$ and the normalised indentation depth $H / R$, as shown in Figure 9(b). Figure 9(a)

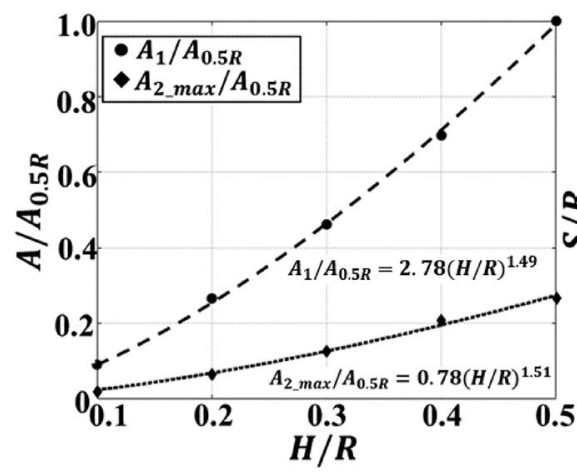

(a)

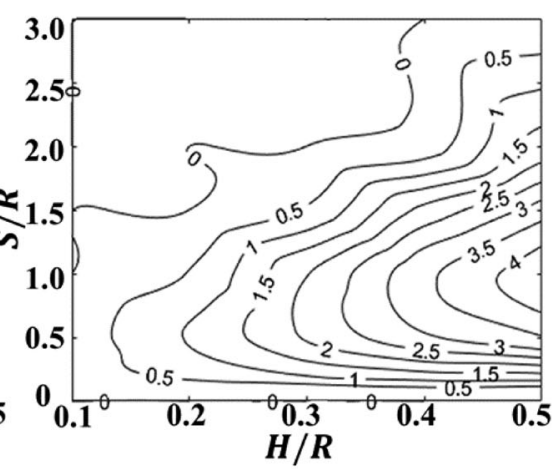

(b)

Figure 9. Normalised size of the deformation-affected area with respect to (a) $H / R$ for single and sequential indentation and (b) $H / R$ and $S / R$ for sequential indentation. 
shows the normalised maximum size of the overlap region $A_{2_{-} \max } / A_{0.5 R}$ for each value of normalised indentation depth $H / R$ across all values of normalised indentation spacing $S / R$. It should be noted that $A_{2-\max } / A_{0.5 R}$ was approximately $0.3 A_{1} / A_{0.5 R}$ for all values of $H / R$. From the figure, these normalised areas followed a relationship in the form: $A / A_{0.5 R}=C(H / R)^{n}$, where $(C, n)=(2.78,1.49)$ for $A_{1}$ and $(C, n)=(0.78,1.51)$ for $A_{2_{-} \max }$. The $R$ squared value for these fits were both 0.99 .

A commonly used metric for mechanical surface treatment methods has come in the form of surface coverage, which has been used to quantify the relative size of the deformed surface area [28,29], as opposed that of the subsurface as in the present work. Prior work has established the importance of control of surface coverage to control overall functional properties by mitigation of possible defects, such as surface cracks or folds at regions undergoing significant deformation [30]. Figure 10 shows a schematic summarising the surface coverage parameter for a single indentation as a function of the indentation spacing $S$ and the projected contact length $L$, this given by $L=2 \sqrt{R^{2}-(R-H)^{2}}$ as in Figure 1. From the figure, several modes of surface coverage are identified. First, when $S<\sqrt{2} L$ as shown in Figure 10(a), the indentation overlaps with pre-strained regions and the target surface is fully covered by surface indentations, this yield surface coverage exceeding $100 \%$. When $\sqrt{2} L \leq S \leq 2 L$ as shown in Figure 10(b), surface coverage drops below $100 \%$ as well as the overlap with the pre-strained regions. Finally, when $S>2 L$ in Figure 10(c), no overlap occurs between successive indents. It is worthwhile to note that the actual deformed surface profile from a single indentation may result in material piling-up or sinking-in at the surface of the indent [31]. In this regard, sinking-in phenomena decreases the actual contact length of $L$ compared to the derived value.

The relationship of surface coverage to measures for quantifying severity and homogeneity of the subsurface deformation can be considered by evaluating the role of the normalised indentation spacing parameter $S / L$ on the maximum

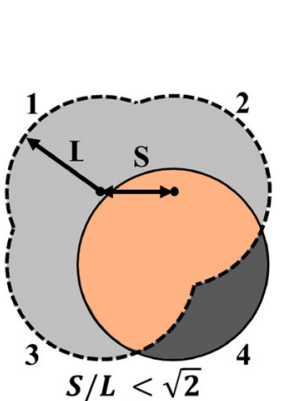

(a)

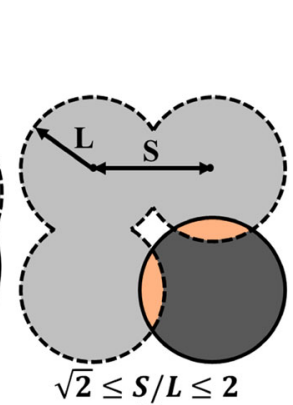

(b)

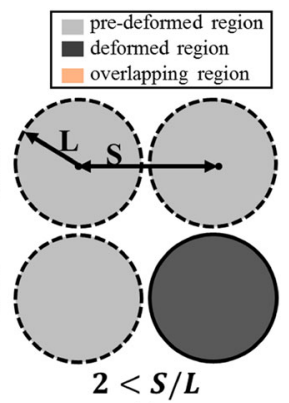

(c)

Figure 10. Schematic of surface coverage dependent on $L$ and $S$ in sequential indentation configuration where (a) $S<\sqrt{2} L$, (b) $\sqrt{2} L \leq S \leq 2 L$ and (c) $S>2 L$. 
strain in the deformed subsurface and the subsurface overlap/coverage. Figure 11(a) shows the ratio of maximum plastic strain $\bar{\varepsilon}_{2_{-} \max } / \bar{\varepsilon}_{1 \_\max }$ with respect to $S / L$. From the figure, at $S / L=0, \bar{\varepsilon}_{2_{-} \max } / \bar{\varepsilon}_{1-\max }=1.0$. This increases to a maximum value of $\bar{\varepsilon}_{2_{-} \max } / \bar{\varepsilon}_{1_{-} \max }=2.5$ at $S / L=1.2$ and decreases to $\bar{\varepsilon}_{2_{-} \max } / \bar{\varepsilon}_{1 \_\max }=1.0$ at $S / L>4$. In Figure $11(\mathrm{~b})$, a similar trend can be observed for subsurface coverage which reaches a peak value of $A_{2} / A_{1}=0.28$ at $S / L=1.1$. The effect of the indentation spacing parameter $S / L$ on the load applied in the sequential indentation process is shown in Figure 11(c), where $F_{1}$ is the load from the first indent and $F_{2}$ the load from the second indent. From the figure, a maximum value of $F_{2} / F_{1}=1.15$ occurred at $S / L=2.0$ and the ratio approached $F_{2} / F_{1}=1.0$ at larger quantities of $S / L$. It should be noted that decay to $F_{2} / F_{1}=$ 1.0 at large indentation spacing is expected as successive indents can be treated as independent deformation events in the presence of sufficiently large relative spacing. From the above analysis, subsurface coverage and subsurface maximum strain parameters are maximised with $S / L$ in the range of $[1.1,1.2]$ and the required indentation load in this range is approximately equivalent to that of a single indent (e.g. $F_{2}=F_{1}$ ).

The relative efficiency of a surface deformation process can be considered by analysis of maximum strain, surface/subsurface coverage and applied load. To quantify the total energy consumed in deforming the subsurface thorough sequential indentation, the total energy dissipated $W_{t}$ was evaluated for each processing condition and was quantified based on the FE-predicted load trajectory, using $W_{t}=\sum_{i=1}^{2} \underset{i}{F} \mathrm{~d} H_{i}$ [32], where $F_{i}, H_{i}$, and $i$ indicate indentation load, depth, and sequence number, respectively. The normalised total energy, $W_{t} / W_{t_{-} 0.5 R}$, dissipated is summarised in Figure 12 in terms of the normalised maximum plastic strain and the normalised subsurface overlap area, where $W_{t \_0.5 R}$ is the total energy consumed during the single indentation with $H / R$ $=0.5$. As expected, Figure 12(a) shows that larger plastic strains in the deformed subsurface result in a larger energy dissipation for a given depth of indentation. Figure 12(a) also shows that it is possible to achieve a given level of maximum

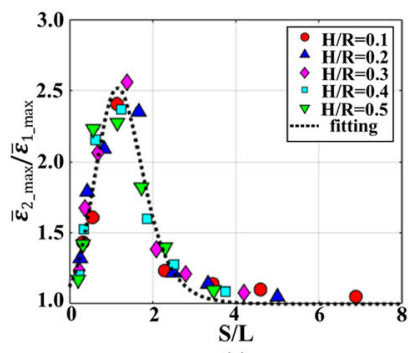

(a)

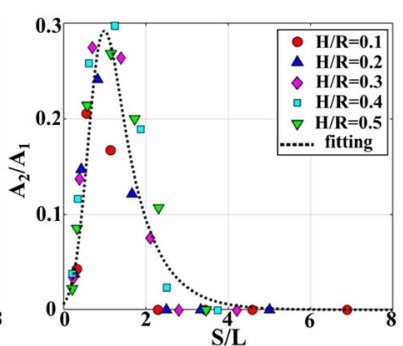

(b)

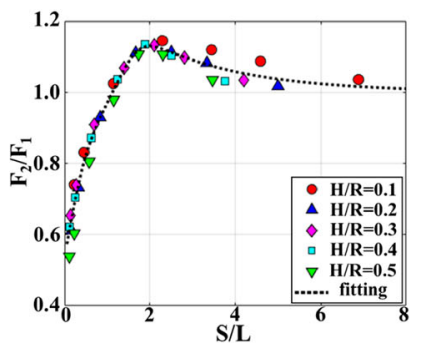

(c)

Figure 11. Ratio of (a) normalised maximum plastic strain, (b) normalised deformation-affected area, and (c) normalised indentation load in sequential indentation with respect to $S / L$ for all investigated conditions. 
plastic strain at lower dissipated energy depending on the sequential indentation parameters. For example, it is possible to achieve a normalised maximum plastic strain of $\bar{\varepsilon}_{2 \_\max } / \bar{\varepsilon}_{0.5 R} \sim 0.2$ at conditions of $[H / R, \mathrm{~S} / \mathrm{R}]=[0.1,0.5]$ and $[H / R$, $\mathrm{S} / \mathrm{R}]=[0.5,0.1]$, where the relative normalised total energy used in the latter condition is $80 \%$ lower. The upper and lower bounds for the total energy dissipated at a given level of strain are marked at the extents of the plot and fit according to an exponential function in the form: $W_{t} / W_{t_{-} 0.5 R}=C\left(\bar{\varepsilon}_{2_{-} \max } / \bar{\varepsilon}_{0.5 R}\right)^{n}$, where the upper bound is defined by $(C, n)=(1.01,2.31)$ and the lower bound defined by $(C, n)=(0.20,2.30)$. Similarly, upper and lower bounds for the subsurface overlap are shown in Figure 12(b) and are given by: $W_{t} / W_{t_{-} 0.5 R}=C\left(A_{2} / A_{0.5 R}\right)^{n}$, where the upper bound is defined by $(C, n)=(39.81,1.02)$ and the lower bound defined by $(C, n)=(7.81,1.08)$.

The present results also have utility for understanding the role of sequential indentation parameters on strain path changes in the deformed subsurface. In this regard, it has been shown that the rate of microstructure refinement in $S^{2} P D$ is sensitive to strain path changes occurring in the subsurface [33]. The influence of strain path changes in accelerating rates of microstructure evolution with respect to strains imposed has been well recognised in other severe plastic deformation (SPD) processes. For instance, the rate of grain refinement in pure $\mathrm{Al}$ alloys was seen to be accelerated in the B-route of equal channel angular pressing (ECAP), this compared to the other routes in ECAP undergoing the same deformation due to presence of strain path changes [33]. Similar effects have been seen in periodic shear-based transient surface generation, where strain path changes coupled with larger pre-strains resulted in greater reductions in grain size [1, 34]. Figure 13(a) shows the evolution of maximum effective plastic strain with a normalised indentation spacing of $S / R=1.0$ and normalised indentation depths of $H / R=[0.2,0.3]$. Plastic strains induced at the first

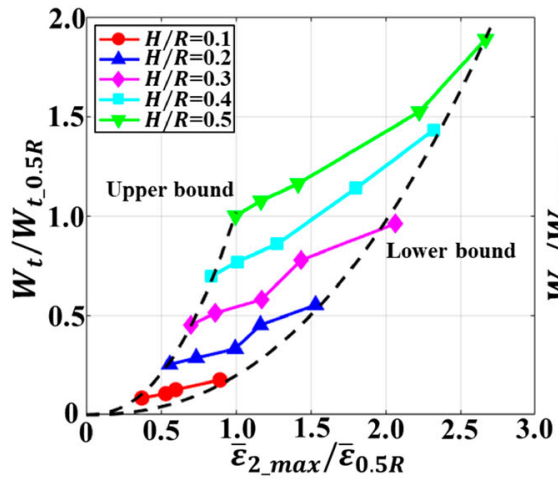

(a)

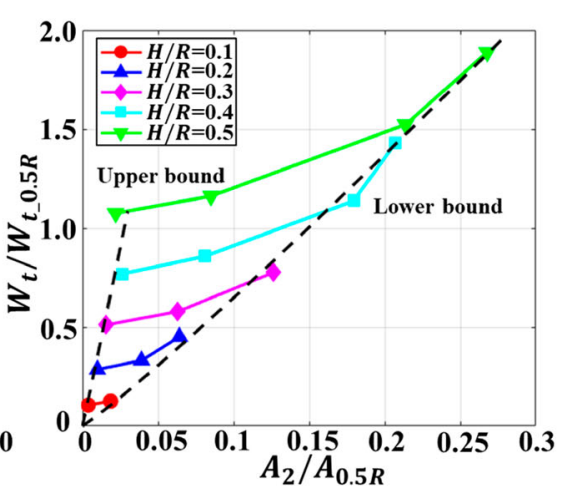

(b)

Figure 12. Normalised total energy with respect to (a) maximum effective plastic strain and (b) maximum deformation-affected area in sequential indentation, where dashed lines indicate the upper and lower bound of total energy. 


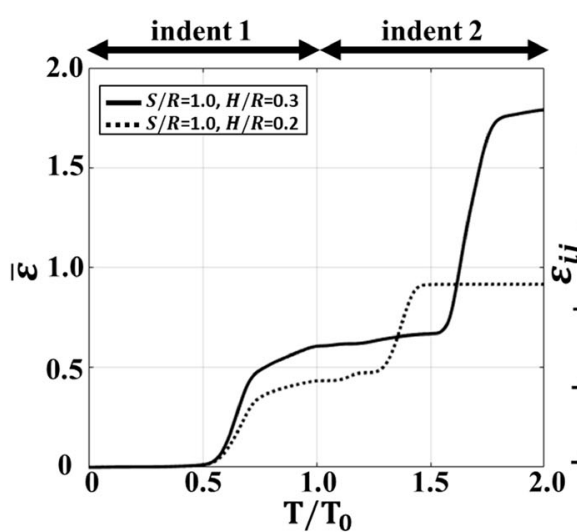

(a)

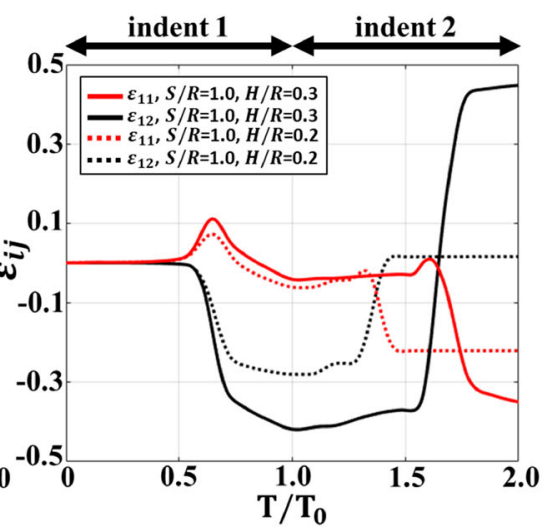

(b)

Figure 13. Evolution of (a) effective plastic strain and (b) Lagrangian strain components with respect to normalised processing time of $T / T_{0}$ in sequential indentation.

indentation were $\bar{\varepsilon}_{1}=[0.47,0.61]$ with $H / R=[0.2,0.3]$. The evolution of each strain component is depicted in Figure 13(b), where $\varepsilon_{22}$ is omitted since $\varepsilon_{22}=-\varepsilon_{11}$ in plane strain. Experimental measures of strain path changes during the two indentations were $\left[\Delta \varepsilon_{11}, \Delta \varepsilon_{12}\right]=[-0.16,0.3]$ with $H / R=0.2$ and $\left[\Delta \varepsilon_{11}, \Delta \varepsilon_{12}\right]=[-0.31,0.88]$ with $H / R=0.3$. Knowledge of these strain path change magnitudes is important since control of these components in surface deformation is critical in controlling texture formation and also in controlling the rate of microstructure refinement at the surface.

\section{Conclusion}

In the present study, the deformation field and load response during sequential indentation of a model strain hardening material was investigated. In-situ observations of the evolving subsurface deformation field were made using DIC-based measurement and these results were found to agree well with both the analytical model and FE-based predictions of the strain distribution and load response. Maximum strain and the degree of strain field overlap in the deformed subsurface were maximised when the ratio of indentation spacing to projected indentation contact length was approximately $S / L=[1.1$, 1.2]. Additionally, relative differences in energy expended were found for differing conditions that produce similar levels of subsurface plastic strain and strain field overlap. In this regard, a lower bound for the maximisation of both parameters can be determined across a range of conditions that include indentation depth and indentation spacing. The role of these parameters on affecting the magnitude of strain path changes occurring in the deformed subsurface were also evaluated in the context of understanding potential effects on microstructure and texture evolution in mechanical surface treatment methods. 


\section{Disclosure statement}

No potential conflict of interest was reported by the authors.

\section{Funding}

The authors acknowledge the financial support by Division of Civil, Mechanical and Manufacturing Innovation [grant number 1254818/1464853] and Third Wave Systems [via DOE subcontract DE-EE0005762].

\section{References}

[1] S. Basu, Z. Wang, R. Liu and C. Saldana, Enhanced subsurface grain refinement during transient shear-based surface generation, Acta Mater. 116 (2016), pp. 114-123.

[2] Y.S. Zhang, Z. Han, K. Wang and K. Lu, Friction and wear behaviors of nanocrystalline surface layer of pure copper, Wear 260 (2006), pp. 942-948.

[3] H.W. Huang, Z.B. Wang, J. Lu and K. Lu, Fatigue behaviors of AISI 316L stainless steel with a gradient nanostructured surface layer, Acta Mater. 87 (2015), pp. 150-160.

[4] L.L. Shaw, J.W. Tian, A.L. Ortiz, K. Dai, J.C. Villegas, P.K. Liaw, R. Ren and D.L. Klarstrom, A direct comparison in the fatigue resistance enhanced by surface severe plastic deformation and shot peening in a C-2000 superalloy, Mater. Sci. Eng. A 527 (2010), pp. 986-994.

[5] Z. Pu, S. Yang, G.L. Song, O.W. Dillon, D.A. Puleo and I.S. Jawahir, Ultrafine-grained surface layer on $\mathrm{Mg}-\mathrm{Al}-\mathrm{Zn}$ alloy produced by cryogenic burnishing for enhanced corrosion resistance, Scr. Mater 65 (2011), pp. 520-523.

[6] B.N. Mordyuk, O.P. Karasevskaya, G.I. Prokopenko and N.I. Khripta, Ultrafine-grained textured surface layer on $\mathrm{Zr}-1 \% \mathrm{Nb}$ alloy produced by ultrasonic impact peening for enhanced corrosion resistance, Surf. Coatings Technol 210 (2012), pp. 54-61.

[7] K. Lu, Making strong nanomaterials ductile with gradients, Science (80-.) 345 (2014), pp. 1455-1456.

[8] X. Wu, P. Jiang, L. Chen, F. Yuan and Y.T. Zhu, Extraordinary strain hardening by gradient structure, Proc. Natl. Acad. Sci 111 (2014), pp. 7197-7201.

[9] N.R. Tao, M.L. Sui, J. Lu and K. Lua, Surface nanocrystallization of iron induced by ultrasonic shot peening, Nanostructured Mater 11 (1999), pp. 433-440.

[10] H.L. Chan, H.H. Ruan, A.Y. Chen and J. Lu, Optimization of the strain rate to achieve exceptional mechanical properties of 304 stainless steel using high speed ultrasonic surface mechanical attrition treatment, Acta Mater. 58 (2010), pp. 5086-5096.

[11] X. Dai, J. Boll, M.E. Hayes and D.E. Aston, Adhesion of Cryptosporidium parvum and Giardia lamblia to solid surfaces: The role of surface charge and hydrophobicity, Colloid. Surf. B 34 (2004), pp. 259-263.

[12] C. Bernal, A.M. Camacho, J.M. Arenas and E.M. Rubio Alvir, Analytical procedure for geometrical evaluation of flat surfaces formed by multiple indentation processes, Appl. Mech. Mater 217-219 (2012), pp. 2351-2356.

[13] S. Bagherifard, R. Ghelichi and M. Guagliano, A numerical model of severe shot peening (SSP) to predict the generation of a nanostructured surface layer of material, Surf. Coatings Technol 204 (2010), pp. 4081-4090.

[14] L.E. Samuels and T.O. Mulhearn, An experimental investigation of the deformed zone associated with indentation hardness impressions, J. Mech. Phys. Solids 5 (1957), pp. $125-134$ 
[15] S.A. Meguid and M.S. Klair, An examination of the relevance of co-indentation studies to incomplete coverage in shot-peening using the finite-element method, J. Mech. Work. Technol 11 (1985), pp. 87-104.

[16] S.A. Meguid, G. Shagal and J.C. Stranart, 3D FE analysis of peening of strain-rate sensitive materials using multiple impingement model, Int. J. Impact Eng 27 (2002), pp. 119-134.

[17] G.H. Majzoobi, R. Azizi and A. Alavi Nia, A three-dimensional simulation of shot peening process using multiple shot impacts, J. Mater. Process. Technol 164-165 (2005), pp. 1226-1234.

[18] C. Bernal, A.M. Camacho, M.M. Marín and B. de Agustina, Methodology for the evaluation of $3 D$ surface topography in multiple indentation processes, Int. J. Adv. Manuf. Technol 69 (2013), pp. 2091-2098.

[19] Z. Wang, S. Basu, T.G. Murthy and C. Saldana, Modified cavity expansion formulation for circular indentation and experimental validation, Int. J. Solids Struct 97-98 (2016), pp. 129-136.

[20] S. Yadav, C. Saldana and T.G. Murthy, Deformation field evolution in indentation of a porous brittle solid, Int. J. Solids Struct 66 (2015), pp. 35-45.

[21] T.G. Murthy, C. Saldana, M. Hudspeth and R. M'Saoubi, Deformation field heterogeneity in punch indentation, Proc. R. Soc. A Math. Phys. Eng. Sci 470 (2014), pp. 20130807-20130807.

[22] N. Sundaram, Y. Guo, T.G. Murthy, C. Saldana and S. Chandrasekar, Rotation field in wedge indentation of metals, J. Mater. Res 27 (2012), pp. 284-293.

[23] T.G. Murthy, C. Huang and S. Chandrasekar, Characterization of deformation field in plane-strain indentation of metals, J. Phys. D. Appl. Phys 41 (2008), p. 074026.

[24] S.K. Kang, Y.C. Kim, K.H. Kim, J.Y. Kim and D. Kwon, Extended expanding cavity model for measurement of flow properties using instrumented spherical indentation, Int. J. Plast 49 (2013), pp. 1-15.

[25] N. McCormick and J. Lord, Digital image correlation, Mater. Today 13 (2010), pp. $52-54$.

[26] S. Basu, Z. Wang and C. Saldana, Deformation heterogeneity and texture in surface severe plastic deformation of copper, Proc. R. Soc. London A Math. Phys. Eng. Sci 472 (2016), pp. 20150486.

[27] Z. Wang, S. Basu, T.G. Murthy and C. Saldana, Gradient microstructure and texture in wedge-based severe plastic burnishing of copper, J. Mater. Res 33 (2018), pp. 10461056.

[28] G.H. Farrahi, J.L. Lebrijn and D. Couratin, Effect of shot peening on residual stress and fatigue life of a Spring steel, Fatigue Fract. Eng. Mater. Struct 18 (1995), pp. 211-220.

[29] C. Guo, Z. Wang, D. Wang and S. Hu, Numerical analysis of the residual stress in ultrasonic impact treatment process with single-impact and two-impact models, Appl. Surf. Sci 347 (2015), pp. 596-601.

[30] S. Bagherifard, I. Fernandez-Pariente, R. Ghelichi and M. Guagliano, Effect of severe shot peening on microstructure and fatigue strength of cast iron, Int. J. Fatigue 65 (2014), pp. 64-70.

[31] X. Hernot and O. Bartier, An expanding cavity model incorporating pile-up and sink-in effects, J. Mater. Res 27 (2012), pp. 132-140.

[32] G.M. Pharr, An improved technique for determining hardness and elastic modulus using load and displacement sensing indentation experiments, J. Mater. Res 7 (1992), pp. 1564-1583.

[33] R.B. Figueiredo, I.P. Pinheiro, M.T.P. Aguilar, P.J. Modenesi and P.R. Cetlin, The finite element analysis of equal channel angular pressing (ECAP) considering the strain path 
dependence of the work hardening of metals, J. Mater. Process. Technol 180 (2006), pp. 30-36.

[34] S. Basu, Z. Wang and C. Saldana, Anomalous evolution of microstructure and crystallographic texture during indentation, Acta Mater. 105 (2016), pp. 25-34. 